OPEN ACCESS

Edited by:

Sukhwan Yoon,

Korea Advanced Institute of Science and Technology, South Korea

Reviewed by:

Hui Li,

Jinan University, China

Baogang Zhang,

China University of Geosciences,

China

*Correspondence:

Kai Li

likai_sino@sina.com

Simon Ming-Yuen Lee

simonlee@um.edu.mo

Specialty section:

This article was submitted to Microbiological Chemistry

and Geomicrobiology,

a section of the journal

Frontiers in Microbiology

Received: 14 April 2020

Accepted: 12 June 2020

Published: 15 July 2020

Citation:

Hu D, Sun C, Jin T, Fan G, Mok KM, Li K and Lee SM-Y (2020)

Exploring the Potential of Antibiotic Production From Rare Actinobacteria by Whole-Genome Sequencing and Guided MS/MS Analysis.

Front. Microbiol. 11:1540. doi: 10.3389/fmicb.2020.01540

\section{Exploring the Potential of Antibiotic Production From Rare Actinobacteria by Whole-Genome Sequencing and Guided MS/MS Analysis}

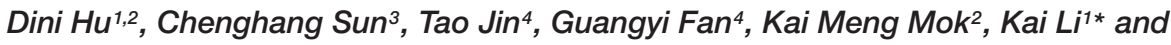 \\ Simon Ming-Yuen Lee ${ }^{*}$ \\ 'School of Ecology and Nature Conservation, Beijing Forestry University, Beijing, China, ${ }^{2}$ Department of Civil \\ and Environmental Engineering, Faculty of Science and Technology, University of Macau, Macau, China, ${ }^{3}$ Institute \\ of Medicinal Biotechnology, Chinese Academy of Medical Sciences and Peking Union Medical College, Beijing, China, \\ ${ }^{4}$ Beijing Genomics Institute, Shenzhen, China, ${ }^{5}$ State Key Laboratory of Quality Research in Chinese Medicine, Institute \\ of Chinese Medical Sciences, University of Macau, Macau, China
}

Actinobacteria are well recognized for their production of structurally diverse bioactive secondary metabolites, but the rare actinobacterial genera have been underexploited for such potential. To search for new sources of active compounds, an experiment combining genomic analysis and tandem mass spectrometry (MS/MS) screening was designed to isolate and characterize actinobacterial strains from a mangrove environment in Macau. Fourteen actinobacterial strains were isolated from the collected samples. Partial 16S sequences indicated that they were from six genera, including Brevibacterium, Curtobacterium, Kineococcus, Micromonospora, Mycobacterium, and Streptomyces. The isolate sp.01 showing $99.28 \%$ sequence similarity with a reference rare actinobacterial species Micromonospora aurantiaca ATCC $27029^{\top}$ was selected for whole genome sequencing. Organization of its gene clusters for secondary metabolite biosynthesis revealed 21 clusters encoded to antibiotic production, which is higher than other Micromonospora species. Of the genome-predicted antibiotics, kanamycin was found through guided MS/MS analysis producible by the $M$. aurantiaca strain for the first time. The present study highlighted that genomic analysis combined with MS/MS screening is a promising method to discover potential of antibiotic production from rare actinobacteria.

Keywords: rare actinobacteria, antibiotics, mangroves, whole-genome sequencing, mass spectrometry

\section{INTRODUCTION}

Microorganisms such as actinobacteria are major sources of antibiotic compounds (Sanchez and Demain, 2015). Actinobacteria are gram-positive bacteria with high GC content in their DNA. They are prolific sources of many well-known antibiotics such as erythromycin, kanamycin, streptomycin, tetracycline, and vancomycin (Schatz et al., 1944; Umezawa et al., 1957; Darken et al., 1960; Cortes et al., 1990; Jung et al., 2007). Among which, nearly 70-80\% of the substances are derived from the genus Streptomyces (Raja and Prabakarana, 2011). Meanwhile, the nonStreptomyces group or rare actinobacteria are lesser known for production of bioactive products 
TABLE 1 | Locations and mangrove tree types for sampling.

\begin{tabular}{llll}
\hline Sites & Coordinate & Samples & Sample type \\
\hline $\mathrm{S} 1$ & $22^{\circ} 8^{\prime} 30^{\prime \prime} \mathrm{N} 113^{\circ} 33^{\prime} 11^{\prime \prime} \mathrm{E}$ & $\begin{array}{l}\text { Aegiceras } \\
\text { corniculatum }\end{array}$ & Plant leaves \\
$\mathrm{S} 2$ & $22^{\circ} 8^{\prime} 29^{\prime \prime} \mathrm{N} 113^{\circ} 33^{\prime} 5^{\prime \prime} \mathrm{E}$ & $\begin{array}{l}\text { Aegiceras } \\
\text { corniculatum, } \\
\text { Kandelia candel }\end{array}$ & Plant leaves \\
\hline
\end{tabular}

due to their lower isolation frequency under laboratory culture conditions (Jose and Jebakumar, 2013). However, recent studies showed that rare actinobacteria could produce antibiotics that were not expected from them; e.g., asukamycin and apramycin were found to be produced by a Mycobacterium species (Hu et al., 2019), and rifamorpholines were found to be produced by an Amycolatopsis species (Xiao et al., 2017). Thus, the antibioticproducing potential of the rare actinobacteria is underexplored, and it is not surprising that they are gaining attention (Tiwari and Gupta, 2012; Dhakal et al., 2017; Ishii, 2019).

The antibiotic-producing ability of an actinobacteria is determined by the biosynthetic gene clusters within its genome (Finan, 2017). With the development of molecular sequencing technology, whole genome sequences could provide a new perspective on discovering the potential of antibiotic production from these microbes (Wright, 2019). A microbial genome usually contains 20 to 40 types of biosynthetic gene clusters, each responsible for producing one compound. For example, the widely exploited Streptomyces avermitilis harbored 25 gene clusters related to production of type-I polyketide compounds, type-II polyketide-derived compounds, and nonribosomal peptide synthetases (Ômura et al., 2001; Ikeda et al., 2003). Bacteria with large number of gene clusters within the genome have high potential in synthesizing multiple types of compounds (Challis, 2014). It is known that microbes can exchange genetic information with each other through the process of horizontal gene transfer (Koonin, 2016). A bacteria species habituating in a diverse and rich microbial community therefore has high possibility of having a larger number of gene clusters in its genome (Cooper et al., 2017). A Streptomyces parvulus species derived from a mangrove zone was found to have 109 gene clusters, many more than those grown in other natural environments ( $\mathrm{Hu}$ et al., 2018). Mangroves are known as productive inter-tidal ecosystems with rich microbial communities, having bacteria making up $91 \%$ of their total microbial biomass (Azman et al., 2015). Their locations being at the transition area between terrestrial and marine habitats give mangroves the advantage of harboring very diverse bacterial communities from two different environments, providing conditions for microbes to exchange genetic information with each other. Thus, mangrove-derived microbes could have greater genetic potential to synthesize more bioactive substances.

Even when genomic information of a bacterial species is available, many of its gene clusters are expressed only weakly or even silent under laboratory fermentation conditions. To ascertain which secondary metabolites can actually be produced by the tested strain, chemical analysis is necessary. The advancement of instrumentation for analytical chemistry has
TABLE 2 | The endophytic actinobacterial community identified based on the partial 16S rRNA sequences.

\begin{tabular}{llcc}
\hline Isolate & Top-hit taxon at species level & Length (bp) & Similarity (\%) \\
\hline 01 & Micromonospora aurantiaca ATCC 27029 $^{\top}$ & 942 & 99.28 \\
02 & Streptomyces cuspidosporus NBRC 12378 & 1005 & 98.28 \\
03 & Streptomyces ederensis NBRC 15410 & 930 & 98.79 \\
04 & Streptomyces hyderabadensis OU-40 & 1001 & 99.18 \\
05 & Streptomyces olivaceus NRRL B-3009 & 1005 & 99.25 \\
06 & Streptomyces pactum NBRC 13433 & 930 & 99.57 \\
07 & Streptomyces parvulus NBRC 13193 & \\
08 & Microbacterium hydrothermale 0704C9-2 & 1047 & 99.13 \\
09 & Microbacterium hydrothermale 0704C9-2 & 1045 & 98.16 \\
10 & Mycobacterium saopaulense EPM 10906 & 1045 & 98.58 \\
11 & Kineococcus aurantiacus IFO 15268 & 1504 & 99.90 \\
12 & Kineococcus mangrove L2-1-L1 & 972 & 98.20 \\
13 & Brevibacterium sediminis FXJ8.269 & 1048 & 98.00 \\
14 & Micrococcus yunnanensis YIM 65004 & 1005 & 98.90 \\
\hline
\end{tabular}

speeded up the exploration of microbial-derived secondary metabolites. For instance, MALDI-TOF-MS was used to identify 10 secondary metabolites related to previously uncharacterized gene clusters in Streptomyces hygroscopicus (Kersten et al., 2011), ESI-IT-MS was applied to uncover a compound of strong antibacterial activity produced by Gliocladium sp. (Koolen et al., 2012), and a Q-Trap LC-MS platform was utilized to unravel natural products with antimicrobial activity produced by Vibrio cholerae (Sikora et al., 2018). Therefore, the coupled method of genome mining and mass spectrometry could reveal the antibiotic production potential of isolated microbes.

The present study is to explore the potential of antibiotic production of a rare actinobacteria from a mangrove environment. Actinobacterial strains were first isolated from collected plant samples by plate culturing and identified by $16 \mathrm{~S}$ sequence analysis. Whole-genome sequencing and genomic analysis of a selected strain were performed to identify the gene clusters encoding antibiotic production. Preliminary tandem mass spectrometry (MS/MS) analysis was then used to identify the small molecules produced from the tested strain under laboratory conditions. The results could provide reference information for exploring new sources of antibiotics for further research and application in the medical industry.

\section{MATERIALS AND METHODS}

\section{Environmental Sampling}

Plant samples from Aegiceras corniculatum and Kandelia candel mangrove trees were collected at two sites, S1 and S2, of different environmental characteristics in the Cotai City Ecological Reserve in Macau during March and May of 2017 as listed in Table 1. Samples of $K$. candel were only collected from site S2 due to its unavailability at site $S 1$. Several leaves were picked from different parts of one tree and put into a sterile plastic bag as a sample. All specimens were transported back to the laboratory immediately from site. 


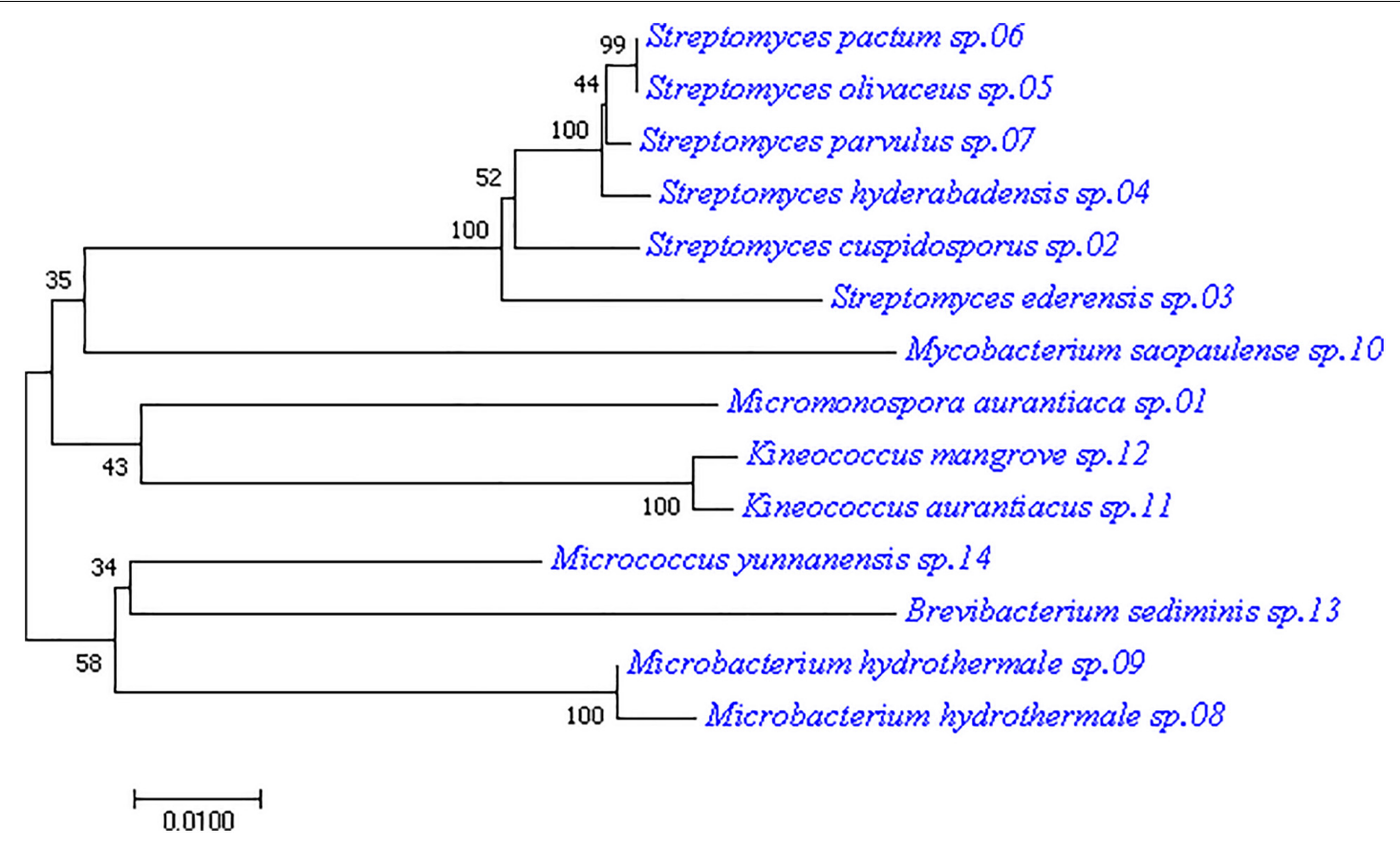

FIGURE 1 | Phylogenetic tree of the 14 actinobacterial strains isolated from mangrove leaves. The tree was constructed with the neighbor-joining method having the number of bootstrap replications set to 1000 .

\section{Selective Isolation of Actinobacteria Strains}

The procedures for plant sample pretreatment and plate culture followed those in $\mathrm{Hu}$ et al. (2018). In short, Tween-20, $\mathrm{NaClO}$, $\mathrm{NaS}_{2} \mathrm{O}_{3}$, ethanol, and $\mathrm{NaHCO}_{3}$ were used to clean the surfaces of the plant samples. Their homogenates were prepared and then diluted to plate onto isolation media. Seven different media including ISP media 2, ISP media 4, ISP media 7, Gauze No. 1, Nutrient Agar, halothiobacillus HL2, and Czapek were used to culture actinobacterial strains. The culture media were incubated at $28^{\circ} \mathrm{C}$ for $7-30$ days.

\section{Extraction of DNA From Pure Cultures and PCR Amplification of 16S rRNA}

DNA was extracted from each purified isolate for bacterial species identification. The procedures for DNA extraction and PCR amplification followed those in $\mathrm{Hu}$ et al. (2018). The universal bacteria primer pair of 27F (5' -AGAGTTTGATCCTGGCTCAG$\left.3^{\prime}\right)$ and 1492R (5'-TACGGCTACCTTGTTACGACTT-3') were used for amplification of $16 \mathrm{~S}$ rRNA sequences (Mao et al., 2012). The yielded DNA was then sequenced on the Sanger sequencing platform. The generated sequences were compared with EzBioCloud database to identify the species ${ }^{1}$.

\section{Genomic Analysis}

The procedures for whole genome sequencing and genomic analysis followed those in $\mathrm{Hu}$ et al. (2018). Genomic DNA was

${ }^{1}$ https://www.ezbiocloud.net/ prepared using the TIANamp Bacteria DNA Kit (TIANGEN Biotech Co. Ltd). The genomic DNA library was constructed using the NEBNext Ultra II DNA Library Prep Kit for sequencing on Illumina NovaSeq HiSeq 4000. Genome assembly and gene prediction were performed by IBDA and MetaGeneMark, respectively. The tRNAscan-SE was used for prediction of ribosomal RNAs (rRNAs). After searching against the Kyoto Encyclopedia of Genes and Genomes (KEGG) database, the functional categories were assigned through the genome. Anti-SMASH (version 4.0 and 5.0) was used to predict the biosynthetic gene clusters for production of secondary metabolites $^{2}$. A threshold of homology similarity at $15 \%$ was chosen for analysis based on previous studies (Huang et al., 2016; Othoum et al., 2019) that found that gene clusters with low similarity could contain unique structures. Mega 7.0 was used to construct the phylogenetic tree. The sequence of Micromonospora aurantiaca sp.01 generated in this study has been deposited in the GenBank database with the accession number RIBT00000000.

\section{Crude Extract Preparation and Mass Spectrometric Analysis}

The procedures for biochemical screening followed those in $\mathrm{Hu}$ et al. (2018). The 4000 Q TRAP LC/MS/MS mass spectrometer system was equipped with a micro-ESI-MS for separation and analysis of the secondary metabolites produced by a targeted strain. Full-scan MS data were acquired from mass-to-charge ratios $(\mathrm{m} / \mathrm{z})$ between 100 and 1000 at an acquisition rate of $0.6 \mathrm{~s}$ per spectrum. ESI source was operated in the positive mode at

\footnotetext{
${ }^{2}$ https://antismash.secondarymetabolites.org/\#!/about
} 


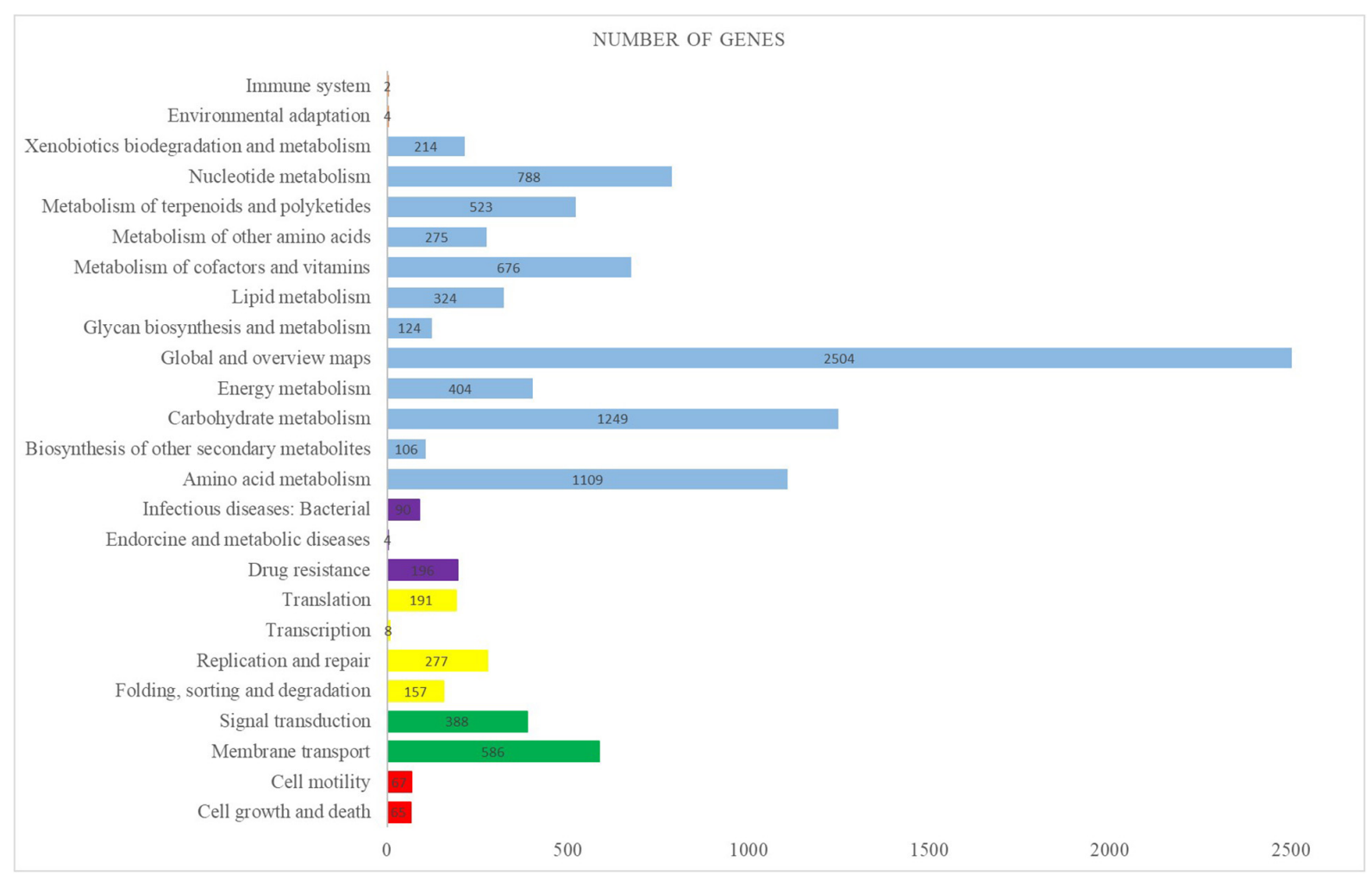

FIGURE 2 | Distribution of KEGG pathways in the Micromonospora aurantiaca sp.01 genome. The distribution of the predicted functional classification proteins was assigned by comparison with the KEGG database. Figure displays the top five KEGG Orthology (KO) categories of the assigned sequences, including cellular process in red, genetic information processing in yellow, environmental information processing in green, organismal systems in orange, human diseases in purple, and metabolism in blue.

$3.0 \mathrm{kV}$ of capillary voltage and $20 \mathrm{~V}$ of cone voltage. Kanamycin standard sulfate salt was purchased from Sigma (St. Louis MO, United States). After drying in vacuum at $70^{\circ} \mathrm{C}$ for $3 \mathrm{~h}$, individual stock solutions were prepared at a concentration of $50 \mathrm{mg} / \mathrm{ml}$ in water (Turnipseed et al., 2009).

\section{RESULTS}

\section{Isolation and Identification of Endophytic Actinobacterial Strains}

Culturable bacteria from the mangrove plant samples were analyzed by plating their pre-treated homogenates onto seven different isolation media. The $16 \mathrm{~S}$ rRNA sequences of the isolated single colonies were amplified by PCR to recover the needed fragments for subsequent Sanger sequencing. The results were then analyzed by using EzbioCloud server referencing with the NCBI database for species identification. The resulting partial 16S sequences revealed 71 endophytic bacterial strains from the plant samples (Supplementary Table S1). Among them, 14 strains were from six genera of the actinobacteria class, including Brevibacterium, Kineococcus, Microbacterium, Micromonospora, Mycobacterium, and Streptomyces. They are listed in Table 2 together with their reference strains and corresponding $16 \mathrm{~S}$ rRNA gene sequence similarity percentages.
Applying the $97.0 \%$ similarity threshold widely used for novel bacterial species identification (Sripreechasak et al., 2013; Kim et al., 2014) as a reference, there were seven isolates considered highly similar to their reference strains (similarity > 99\%), 6 isolates considered closely related to their reference strains (similarity $>98 \%$ ), and 1 isolate considered between closely related to and related to its reference strain (similarity $=98 \%$ ). In terms of genus diversity, Streptomyces was the dominant one harboring six strains, followed by Microbacterium with three strains, and Kineococcus with two strains. Meanwhile, each of the genus Brevibacterium, Micromonospora, and Mycobacterium had 1 strain. A phylogenetic tree was constructed for these 14 actinobacterial isolates to demonstrate their evolutionary phylogenetic relationship (Figure 1).

As only the genus Streptomyces has been exploited extensively for antibiotic production over the years, the underexploited non-Streptomyces or rare actinobacteria group could have much potential not being discovered even for their betterknown genera. Among the rare actinobacteria, the genus Micromonospora has been found predominant in mangroves of different parts of the world over the years (Azman et al., 2015); it was also found in the present study. The phylogenetic tree in Figure 1 showed that isolate $M$. aurantiaca sp.01 was located on the same tree branch of isolate 11 and isolate 12 of the Kineococcus genus, suggesting that they were closely related. It 
TABLE 3 | Overview of the predicted secondary metabolites from biosynthetic gene clusters of the Micromonospora aurantiaca sp.01 detected by anti-SMASH.

\begin{tabular}{|c|c|c|c|c|c|c|}
\hline Type & Location & Category & $\begin{array}{l}\text { Predicted } \\
\text { product }\end{array}$ & $\begin{array}{c}\text { Similarity } \\
(\%)\end{array}$ & Reference strain & $\begin{array}{l}\text { Accession } \\
\text { number }\end{array}$ \\
\hline Type I PKS & $1-5012$ & Antibiotics & Aculeximycin & 23 & Kutzneria albida DSM 43870 & CP007155 \\
\hline Type I PKS & $1-14106$ & & Amphotericin & 23 & Streptomyces nodosus & AF357202 \\
\hline Type I PKS & $1-37928$ & & Chalcomycin & 12 & Streptomyces bikiniensis strain NRRL 2737 & AY509120 \\
\hline Type I PKS & $1-6925$ & & Lobophorin & 13 & Streptomyces sp. SCSIO 01127 & KC013978 \\
\hline Type I PKS & $1-27275$ & & Naphthomycin & 25 & Streptomyces sp. CS & GQ452266 \\
\hline Type I PKS & $1-10526$ & & Naphthomycin & 15 & Streptomyces sp. CS & GQ452266 \\
\hline Type I PKS & $1-10185$ & & Nigericin & 44 & Not derived from GenBank & \\
\hline Type I PKS-NRPS & $57831-125457$ & & Bleomycin & 12 & Streptomyces verticillus & AF210249 \\
\hline Type II PKS & $6402-33702$ & & Kosinostatin & 11 & Micromonospora sp. TP-A0468 & JN038178 \\
\hline $\begin{array}{l}\text { Type II PKS-Fatty } \\
\text { acid }\end{array}$ & $66691-116600$ & & Xantholipin & 16 & Streptomyces flavogriseus strain SIIA-A02191 & GQ421798 \\
\hline $\begin{array}{l}\text { NRPS-Type I } \\
\text { PKS-Siderophore- } \\
\text { Otherks }\end{array}$ & $30808-150128$ & & Azicemicin & 13 & Kibdelosporangium sp. MJ126-NF4 & GU134622 \\
\hline Lantipeptide & $54973-80539$ & & Incednine & 2 & Streptomyces sp. ML694-90F3 & AB767280 \\
\hline Oligosaccharide & $669851-709255$ & & Cosmomycin D & 35 & Streptomyces olindensis strain DAUFPE 56222 & JJOH01000002 \\
\hline Terpene & $118010-138340$ & & Nocathiacin & 4 & Nocardia sp. ATCC 202099 & GU564398 \\
\hline Putative & $120901-132867$ & & Clorobiocin & 14 & Streptomyces roseochromogenes subsp. oscitans & AF329398 \\
\hline Putative & $224076-232740$ & & Fengycin & 20 & Bacillus amyloliquefaciens subsp. plantarum str. FZB42 & CP000560 \\
\hline Putative & $383554-397823$ & & Friulimicin & 6 & Actinoplanes firuliensis & AJ488769 \\
\hline Putative & $121404-131026$ & & Kanamycin & 15 & Streptomyces kanamyceticus & AB254081 \\
\hline Putative & $16035-35841$ & & Lasalocid & 3 & Streptomyces lasaliensis strain NRRL 3382R & FM173265 \\
\hline Putative & $223676-244438$ & & Maklamicin & 8 & Micromonospora sp. GMKU326 & LC021382 \\
\hline Putative & $69108-79942$ & & Pyrrolomycin & 18 & Streptomyces sp. UC 11065 & EF140903 \\
\hline Putative & 454-16503 & & Rifamycin & 15 & Salinispora arenicola CNS-205 & CP000850 \\
\hline Type I PKS & $1-17368$ & $\begin{array}{l}\text { Bioactive } \\
\text { compounds }\end{array}$ & Halstoctacosanolide & 77 & Streptomyces halstedii & AB241068 \\
\hline Type I PKS & $1-5987$ & & Stambomycin & 36 & Streptomyces ambofaciens ATCC 23877 & AM238664 \\
\hline Type I PKS & $169785-215637$ & & Tiancimycin & 19 & Streptomyces sp. CB03234 & KT716443 \\
\hline Fatty acid & 188064-209008 & & Chlorizidine A & 7 & Streptomyces sp. CNH-287 & KF585133 \\
\hline $\begin{array}{l}\text { Oligosaccharide- } \\
\text { Fatty } \\
\text { acid-Terpene- } \\
\text { NRPS }\end{array}$ & $298906-371903$ & & Lobosamide & 13 & Micromonospora sp. RL09-050-HVF-A & KT209587 \\
\hline $\begin{array}{l}\text { Transatpks-NRPS- } \\
\text { Otherks }\end{array}$ & $514354-599495$ & & Leinamycin & 15 & Streptomyces atroolivaceus & AF484556 \\
\hline Putative & $408661-429833$ & & Diazepinomicin & 5 & Micromonospora sp. M42 & KK037233 \\
\hline Type I PKS & $1-6472$ & Others & ECO-02301 & 35 & Streptomyces aizunensis strain NRRL B-11277 & AY899214 \\
\hline Type I PKS & $219687-252289$ & & ECO-02301 & 42 & Streptomyces aizunensis strain NRRL B-11277 & AY899214 \\
\hline Type III PKS & $532-41533$ & & $\begin{array}{l}\text { Alkyl-O- } \\
\text { Dihydrogeranyl- } \\
\text { Methoxyhydroquinones }\end{array}$ & $\begin{array}{r}71 \\
\text { es }\end{array}$ & Actinoplanes missouriensis & AP012319 \\
\hline $\begin{array}{l}\text { Bacteriocin- } \\
\text { Terpene }\end{array}$ & $19400-48982$ & & Lymphostin & 33 & Salinispora tropica CNB-440 & CP000667 \\
\hline Other & $1-10874$ & & Landepoxcin & 11 & Uncultured bacterium AR 412 & KP830093 \\
\hline Saccharide & $144044-173388$ & & Clavulanic acid & 8 & Streptomyces clavuligerus ATCC 27064 & DS570624 \\
\hline Saccharide & $98895-137389$ & & Echosides & 17 & Streptomyces sp. LZ35 & KJ156360 \\
\hline Saccharide & $8423-64174$ & & Phosphonoglycans & 6 & Stackebrandtia nassauensis DSM 44728 & CP001778 \\
\hline Saccharide & $192353-255024$ & & Phosphonoglycans & 12 & Glycomyces sp. NRRL B-16210 & KJ125437 \\
\hline Terpene & $8286-21936$ & & Phosphonoglycans & 3 & Glycomyces sp. NRRL B-16210 & KJ125437 \\
\hline Terpene & $388501-409427$ & & Sioxanthin & 100 & Salinispora tropica CNB-440 & СР000667 \\
\hline Putative & $576-23953$ & & Galbonolides & 10 & Streptomyces galbus strain KCCM 41354 & GU300145 \\
\hline Putative & $11934-29777$ & & Sioxanthin & 100 & Salinispora tropica CNB-440 & CP000667 \\
\hline Type I PKS & $1-5290$ & & & & & \\
\hline
\end{tabular}

(Continued) 
TABLE 3 | Continued

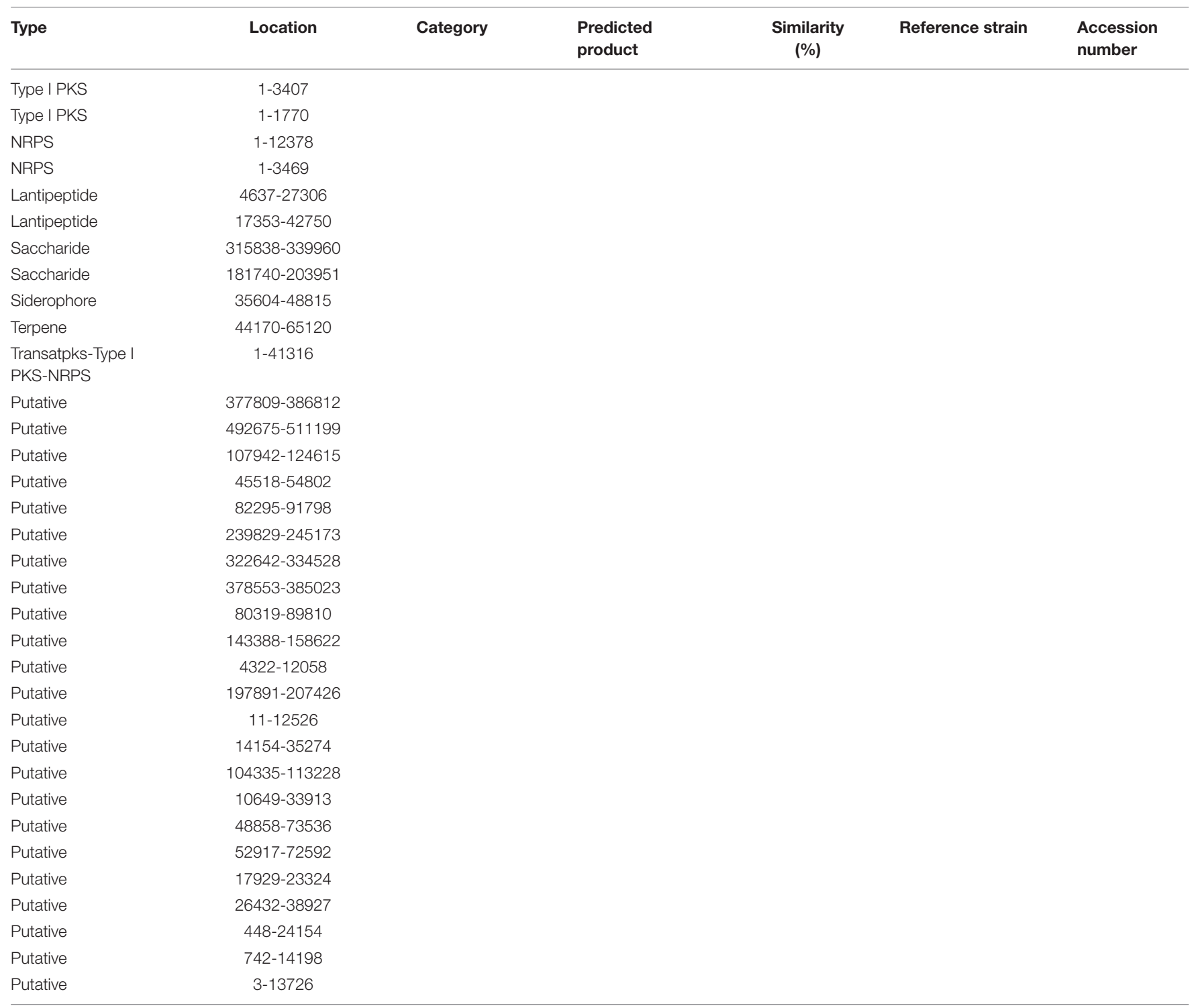

also had close relation with the Streptomyces genus isolates 02, 03, 04, 05, 06, and 07 as well as with the Mycobacterium genus isolate 10. Meanwhile, isolate $M$. aurantiaca sp.01 was found to have farther genetic distances with isolates 08, 09, 13, and 14 of the genera Microbacterium, Brevibacterium, and Micrococcus. Finally, isolate $M$. aurantiaca sp.01 that exhibited $99.28 \% 16 \mathrm{~S}$ similarity with the reference strain M. aurantiaca ATCC $27029^{\mathrm{T}}$ was selected for exploration of its bioactive potential through whole genome sequencing and chemical analysis.

\section{General Feature of the Genome and Associated Secondary Metabolome}

The complete genome sequence of the $M$. aurantiaca sp.01 was deposited to NCBI that produced 16,952,140 reads and 914 scaffolds. The De novo assembly was then done on the yielded reads by using IDBA and generated a total consensus of $8,186,173 \mathrm{bp}$, with an average size of $8956 \mathrm{bp}$ and a
$\mathrm{G}+\mathrm{C}$ content of $72.31 \%$. Gene predictions from the genome of $M$. aurantiaca sp.01 were performed through annotation by comparison with the KEGG database. It showed that a total of 7226 protein-encoding genes were conserved in the genome, 72 tRNA were predicted, the average CDS length was 969 bp, and the coding density was about 89.56\%. The predicted proteins assigned in the KEGG pathways revealed that the top three categories of functional classification were "global and overview maps, carbohydrate metabolism, and amino acid metabolism" (Figure 2).

\section{Antibiotic Biosynthetic Capability of M. aurantiaca sp.01}

AntiSMASH was used to get a preview of the secondary metabolome in the genome of the $M$. aurantiaca sp.01 based on its sequence analysis. Biosynthetic gene clusters encoding for known domains of polyketides (PKS), non-ribosomal 


\section{A Xantholipin-like gene cluster $-\mathrm{A}-\mathrm{KS}-\mathrm{A}=\mathrm{KS}=\mathrm{A}-\mathrm{KS}-$ Polyketide- \\ B Nigericin-like gene cluster $-\mathrm{KS}=\mathrm{AT}=\mathrm{KR}-\mathrm{KR}-$ \\ C Naphthomycin-like gene cluster $=\mathrm{A}-\mathrm{CAL} \cdot \mathrm{KS}=\mathrm{AT}=\mathrm{DH}-\mathrm{KR} \cdot \mathrm{KS}=\mathrm{AT}-\mathrm{DH}=\mathrm{ER}-\mathrm{KR}=\mathrm{OMT} \cdot \mathrm{TE}, \mathrm{KS}=\mathrm{AT}$ \\ D Amphotericin-like gene cluster $-\mathrm{DH}-\mathrm{ER}-\mathrm{KR}-\mathrm{KS}-\mathrm{AT}-\mathrm{KR}-\mathrm{KS}-\mathrm{AT}-\mathrm{KR}-\mathrm{KS}-$ \\ E Aculeximycin-like gene cluster KS AT \\ FIGURE 3 | Proposed antibiotic biosynthetic gene clusters of xantholipin-like (A), nigericin-like (B), naphthomycin-like (C), amphotericin-like (D), and aculeximycin-like (E) compound in the Micromonospora aurantiaca sp.01. Domain notation: A, adenylation; KS, $\beta$-ketoacyl synthase; AT, acyl-CoA/ACP transacylase; DH, dehydratase; TE, thioesterase; KR, ketoreductase; CAL, CoA ligase; ER, enoyl reductase; oMT, O-methyltransferase.}

peptides (NRPS), as well as core and accessory genes were identified by searching individual open reading frames (ORFs) against the sequence database. Nucleotide sequence analysis found 77 gene clusters related to secondary metabolite biosynthesis in the $M$. aurantiaca sp.01 genome. They were predicted to be involved in PKS, NRPS, bacteriocin, fatty acid, lantipeptide, oligosaccharide, saccharide, siderophore, terpene, and many putative products (Table 3). Among them, 42 gene clusters were annotated to 37 known secondary metabolites as antibiotics, bioactive compounds, and other products.

Twenty-one of these 37 secondary metabolites were antibiotics with 10 associated with PKS/NRPS pathways. It is known that secondary metabolite produced through the PKS/NRPS pathway is one of the most important biosynthesis processes involved with the chemical synthesis of biologically active compounds produced by microorganisms (Ansari et al., 2004). Genome analysis of the $M$. aurantiaca sp.01 showed that it contained at least 23 kinds of PKS/NRPS gene clusters, but only 11 of them were found related to known antibiotics.

Furthermore, five of the PKS/NRPS gene clusters with homology similarity greater than $15 \%$ were related to synthesis of antibiotic compounds, namely, aculeximycin, amphotericin, naphthomycin, nigericin, and xantholipin. The xantholipinlike cluster was a hybrid type II PKS-fatty acid gene cluster containing $46 \mathrm{ORFs}$ and 8 catalytic domains, one of which is directly related to polyketide synthetase (Figure 3A). Core biosynthetic genes of this cluster were annotated to cytochrome P450, AMP-dependent synthetase and ligase, beta-ketoacyl synthase, and AraC family transcriptional regulator. Additional biosynthetic genes were related to 8-amino-7-oxononanoate synthase, aminotransferase, alpha/beta hydrolase domaincontaining protein, HAD-superfamily hydrolase, subfamily IA, variant, 1-deoxy-D-xylulose-5-phosphate synthase, transketolase, acyl carrier protein, acetyl-CoA carboxylase biotin carboxylase, carboxyl transferase, polyketide synthesis cyclase, and oxidoreductase. In addition, several transportrelated and regulatory genes can also be found in the genome, including major facilitator transporter, RND family efflux transporter MFP subunit, transcriptional regulator, and LuxR family transcriptional regulator.

Besides the hybrid synthase of PKS-type gene cluster, one of the Type I PKS gene clusters contained six catalytic domains and was responsible for nigericin biosynthesis (Figure 3B). In the genome of $M$. aurantiaca sp.01, only two ORFs of core biosynthetic genes, namely, beta-ketoacyl synthase and malonyl CoA-acyl carrier protein transacylase, were annotated to nigericin biosynthesis. No additional biosynthetic genes were found in the nigericin biosynthetic gene cluster. This cluster displayed $44 \%$ homology to an existing cluster that was not recorded in the GenBank. Thus, it could play an important role in the synthesis of the nigericin-derived primer unit, meaning that these genes could have been transferred horizontally from other microorganisms.

The other three Type I PKS antibiotic biosynthetic gene clusters were naphthomycin-like, amphotericin-like, and aculeximycin-like. The naphthomycin gene cluster showed 25\% homology to that of Streptomyces sp. CS and contained 11 ORFs, in turn encoding 15 catalytic domains related to the polyketide synthetase (Figure 3C). Core and additional biosynthetic genes 
TABLE 4 | Predicted and detected values of $\mathrm{m} / \mathrm{z}$ for the potential secondary metabolites of the Micromonospora aurantiaca sp.01.

\begin{tabular}{|c|c|c|c|}
\hline Category & $\begin{array}{l}\text { Most similar known } \\
\text { cluster }\end{array}$ & $\begin{array}{l}\text { Theoretical } \mathrm{MH}^{+} \\
(\mathrm{m} / \mathrm{z})\end{array}$ & $\begin{array}{l}\text { Detected } \\
\mathrm{MH}^{+}(\mathrm{m} / \mathrm{z})\end{array}$ \\
\hline \multirow[t]{21}{*}{ Antibiotics } & Aculeximycin & 1673.97297 & \\
\hline & Amphotericin & 896.50076 & \\
\hline & Chalcomycin & 701.37484 & \\
\hline & Lobophorin & 1157.63726 & \\
\hline & Naphthomycin & 706.27829 & \\
\hline & Nigericin & 725.48399 & \\
\hline & Bleomycin & 1415.52681 & \\
\hline & Kosinostatin & 632.23700 & \\
\hline & Xantholipin & 552.06975 & \\
\hline & Azicemicin & 460.16076 & \\
\hline & Nocathiacin & 1307.18980 & \\
\hline & Incednine & 738.46934 & \\
\hline & Cosmomycin D & 1189.59070 & \\
\hline & Clorobiocin & 697.21642 & \\
\hline & Fengycin & 1463.80376 & \\
\hline & Friulimicin & 1303.68980 & \\
\hline & Kanamycin & 485.24589 & 485.4 \\
\hline & Lasalocid & 591.38970 & \\
\hline & Maklamicin & 525.32162 & \\
\hline & Pyrrolomycin & 356.91813 & \\
\hline & Rifamycin & 698.31765 & \\
\hline \multirow{7}{*}{$\begin{array}{l}\text { Bioactive } \\
\text { compounds }\end{array}$} & Halstoctacosanolide & 817.51021 & \\
\hline & Stambomycin & $\mathrm{N} / \mathrm{A}$ & \\
\hline & Tiancimycin & 463.12672 & 463.4 \\
\hline & Chlorizidine A & 444.94943 & \\
\hline & Lobosamide & 484.30630 & 484.4 \\
\hline & Leinamycin & 511.10312 & \\
\hline & Diazepinomicin & 463.25968 & 463.4 \\
\hline \multirow[t]{9}{*}{ Others } & ECO-02301 & 1285.79376 & \\
\hline & $\begin{array}{l}\text { Alkyl-O- } \\
\text { Dihydrogeranyl- } \\
\text { Methoxyhydroquinones }\end{array}$ & N/A & \\
\hline & Lymphostin & 311.11442 & \\
\hline & Landepoxcin & $\mathrm{N} / \mathrm{A}$ & \\
\hline & Clavulanic acid & 200.05590 & \\
\hline & Echosides & 467.17060 & \\
\hline & Phosphonoglycans & $\mathrm{N} / \mathrm{A}$ & \\
\hline & Sioxanthin & 727.45738 & \\
\hline & Galbonolides & 381.22772 & \\
\hline
\end{tabular}

were annotated to AMP-dependent synthetase and ligase, 3-dehydroquinate synthase, monooxygenase FAD-binding, methyltransferase, thioesterase, beta-ketoacyl synthase, and malonyl CoA-acyl carrier protein transacylase. The amphotericin gene cluster also had two ORFs encoded to 10 domains (Figure 3D). Compared with the most similar known cluster of Streptomyces nodosus, the amphotericin gene cluster in the studied strain had several unique biosynthetic genes, such as crotonyl-CoA reductase/alcohol dehydrogenase. The last Type I PKS antibiotic synthetic gene cluster was related to aculeximycin synthesis and contained three domains (Figure 3E). Its core biosynthetic genes were annotated for beta-ketoacyl synthase and short-chain dehydrogenase/reductase SDR.

It is worth noting that many observed gene clusters in the M. aurantiaca sp.01 displayed similarity to known clusters of non-Micromonospora strains, meaning that it could probably synthesize many small-molecule compounds through different pathways.

\section{Genome-Guided Natural Product Discovery of Kanamycin}

With the potential antibiotics and bioactive compounds predicted by the genome analysis, secondary metabolites produced by of the $M$. aurantiaca sp.01 under laboratory conditions were examined by reviewing its small-molecule profile for the presence of the predicted compounds. As fermentation and mass spectrometry were used to identify the predicted compounds that are characterized by their $\mathrm{m} / \mathrm{z}, \mathrm{m} / \mathrm{z}$ values of 33 predicted secondary metabolites were first estimated theoretically with ChemDraw as listed in Table 4. As the chemical structures of stambomycin, alkyl-O-dihydrogeranylmethoxyhydroquinones, landepoxcin, and phosphonoglycans are not known, values of their $\mathrm{m} / \mathrm{z}$ could not be estimated and are marked as N/A in Table 4. A 4000 Q TRAP LC/MS/MS system was used to separate the secondary metabolites of the M. aurantiaca sp.01 for analysis. In the first scan mode, four predicted secondary metabolites were detected in the range of $\mathrm{m} / \mathrm{z}$ between 400 and 500 as shown in Table 4. Only one was an antibiotic. The other three were bioactive compounds.

However, only the antibiotic kanamycin at $\mathrm{m} / \mathrm{z}=485.4$ (Figure 4A) could be observed in the second scan mode by its corresponding in-source fragment at $\mathrm{m} / \mathrm{z}=163.4$ (Figure 4B) referencing to the value published in Zhang X. et al. (2019). A standard kanamycin compound was also fed to the 4000 Q TRAP LC/MS/MS system to generate its first scan and second scan spectra that gave its $\mathrm{m} / \mathrm{z}=485.4$ (Figure $4 \mathrm{C}$ ) and $\mathrm{m} / \mathrm{z}=163.4$ (Figure 4D) measurements, respectively. Production of kanamycin by the M. aurantiaca sp. 01 was confirmed.

\section{DISCUSSION}

Microorganisms are prolific sources of diverse bioactive metabolites for many important natural products applied in agricultural, environmental, medical, and industrial areas (Katz and Baltz, 2016), and the actinobacterial group is extremely important for the pharmaceutical sector (Manivasagan et al., 2015). Among the actinobacteria in natural environments, Streptomyces species represent the dominant population; they are commonly found in heavy metal-contaminated soil and their abundances increase with heavy metal stress and the depth of soil (Cao et al., 2017; Zhang et al., 2019b; Wang et al., 2020). As a result, the non-Streptomyces species (or rare actinobacteria) are relatively underexplored and less studied due to their low abundance in taxa (Subramani and Aalbersberg, 2013). In this study, there were 71 bacterial species isolated from the plant samples of a mangrove environment in Macau. There were six genera of actinobacteria found in 14 
isolates; they were Brevibacterium, Kineococcus, Microbacterium, Micromonospora, Mycobacterium, and Streptomyces. It is known that $70-80 \%$ of discovered bioactive compounds are derived from the Streptomyces genus (Qian et al., 2015). Because of this, Streptomyces strains isolated from different environments were repeatedly found to produce similar chemical compounds (Matsumoto and Takahashi, 2017). As a result, the search for useful antibiotics from other sources such as rare actinobacteria for new natural products is of great interest (Demain, 2014). Micromonospora has been reported as a dominant genus of rare actinobacteria in different studied sites (Xu et al., 1996; Ara et al., 2002; Prieto-Davó et al., 2008; Carro et al., 2012), and it was found to produce a commonly used aminoglycoside antibiotic, gentamicin (Weinstein et al., 1963). Meanwhile, several novel natural products such as retymicin, galtamycin $B$, saquayamycin $Z$, and ribofuranosyllumichrome have also been isolated successfully from Micromonospora (Antal et al., 2005). Therefore, the M. aurantiaca sp.01, a rare actinobacterial strain isolated in the present study, was investigated for its biosynthesis potential in depth through whole genome sequencing and mining. Note that no known antibiotic compounds have been reported to be produced by any $M$. aurantiaca strain before (Kim et al., 2006).

Sequenced genomes can provide substantial evidence for the presence of highly diverse secondary metabolic pathways in microorganisms (Bachmann et al., 2014). Gene clusters involved in the synthesis pathways of secondary metabolites in the M. aurantiaca sp.01 were identified by complete sequencing, assembly, and annotation of its genome. Genome mining results showed that the species has the potential to produce 21 antibiotics (Table 4). This predicted potential was considerably greater than those observed in previous studies; it was reported before that only several types of fatty acids and amino acids could be identified in the extracts of M. aurantiaca strains (Dickschat et al., 2011; Xu et al., 2014). A striking feature of the M. aurantiaca sp.01 genome was that it possessed remarkably diverse and abundant PKS biosynthetic pathways; it had 11 PKS gene clusters with similarity over $15 \%$ (Table 3). This number was greater than those in other model actinobacterial genomes, e.g., Salinispora tropica only had only four PKS gene clusters (Udwary et al., 2007), Streptomyces ambofaciens ATCC23877 had 9 (Laureti et al., 2011), and Sorangium cellulosum So ce90 also had 9 (Molnar et al., 2000). Another motivation for sequencing the $M$. aurantiaca sp.01 was that it was considered the most abundant rare actinobacterial genus other than the Streptomyces spp., and no known antibiotic compounds had been reported from any M. aurantiaca strain (Kim et al., 2006). The high genetic potential for secondary metabolite production observed in the $M$. aurantiaca sp.01 genome is encouraging, and more exciting is that most of these predicted antibiotics had never been identified from the Micromonospora genus under fermentation cultivation conditions before. On the other hand, these genome-predicted secondary metabolites had been identified widely from

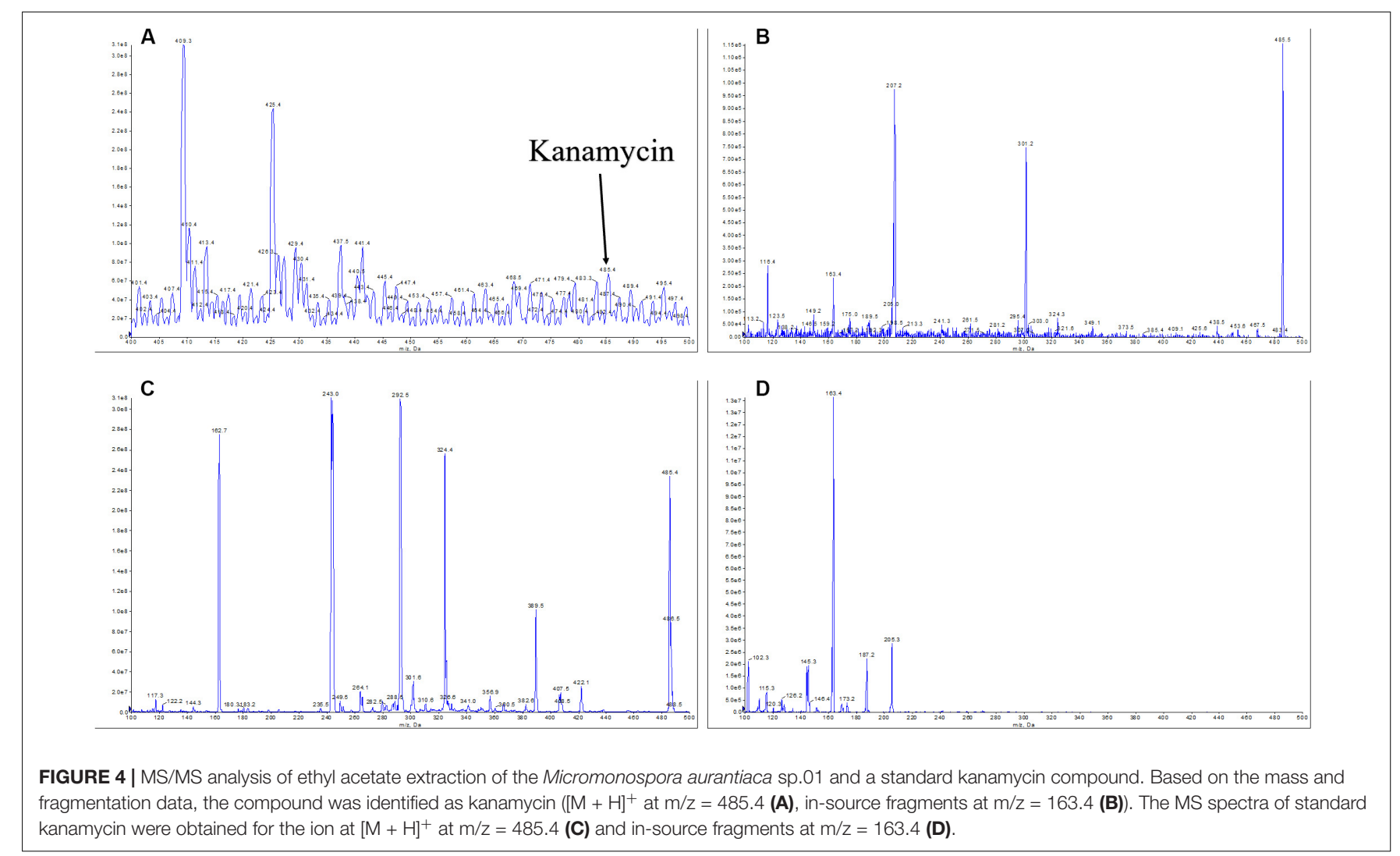


other actinobacterial or non-actinobacterial strains, such as Streptomyces flavogriseus strain SIIA-A02191, Streptomyces sp. CS, S. nodosus, Kutzneria albida DSM 43870, Bacillus amyloliquefaciens subsp. plantarum str. FZB42, Streptomyces sp. UC 11065, Streptomyces kanamyceticus, Salinispora arenicola CNS-205, and Streptomyces olindensis strain DAUFPE 56222 (Table 3). There is no doubt that the genomepredicted secondary metabolite production patterns of the M. aurantiaca sp.01 isolated from mangroves in this study were highly complex.

Mangroves could grow in the harsh conditions of high salinity, extreme tides, strong winds, high temperatures, and muddy anaerobic soils (Ansari et al., 2014). Actinobacterial species such as the $M$. aurantiaca sp.01 of the present study growing in mangroves need to cope with such extreme and biodiverse environment by taking on adaptive strategies that could be reflected in their genome sequences. Previous studies had shown that actinobacterial species in extreme environments had a larger number of genes involved in various types of metabolic pathways when compared with strains from other natural environments (Hu et al., 2018, 2019). Therefore, unexpected abilities in producing novel or more natural products of the actinobacterial strains from extreme environments such as mangroves could be revealed with DNA sequencing technology and genomic analysis.

The assembly, functional annotation, and detailed analysis of the $M$. aurantiaca sp.01 genome sequences were to better understand the biosynthetic potential of a rare actinobacteria. Based on the genome mining results, MS screening was used to look for the predicted secondary metabolites, particularly antibiotics, of the M. aurantiaca sp.01 under laboratory conditions. In general, HPLC can accurately and efficiently separate and quantify each component in a mixture; it does involve tedious development of an appropriate analytical method suitable for an unknown (or a partly known) sample (Zhang et al., 2019a). MS analysis, coupled with MS/MS, is efficient and reliable in identifying unknown compounds without the need for a specific method development, which is more suitable for the present study to rapidly identify the compounds from fermentation products. The MS screening results revealed that the predicted secondary metabolite of kanamycin could be produced. Genomic analysis showed that the kanamycinlike biosynthetic gene cluster of the $M$. aurantiaca sp.01 consisted of 11 ORFs with the core biosynthetic genes encoded to short-chain dehydrogenase/reductase SDR and acyl-CoA dehydrogenase. The finding of kanamycin production in the studied strain was exciting as this antibiotic was first isolated from the S. kanamyceticus species (Umezawa, 1958), and there were no reports on its production by any $M$. aurantiaca species until now.

In addition, using the MS spectrum of the standard kanamycin at $50 \mathrm{mg} / \mathrm{ml}$ mass concentration as a reference (Figures $4 C, D$ ), the estimated kanamycin yield of the $M$. aurantiaca sp.01 cultured in $20 \mathrm{ml}$ of medium with glucose as carbon source for 7 days at $\mathrm{pH} 7.2$ was over $2 \mathrm{mg} / \mathrm{ml}$. Studies indicated that varying the culture conditions, such as $\mathrm{pH}$ value, and carbon and nitrogen source, of the kanamycin producing $S$. kanamyceticus species would vary its yield.
For example, Basak and Majumdar (1973) found that a S. kanamyceticus ATCC 12853 strain cultured in $30 \mathrm{ml}$ of medium with glucose as carbon source for 7 days at $\mathrm{pH}$ 7.0 yielded a $2 \mathrm{mg} / \mathrm{ml}$ kanamycin mass concentration, and then increasing just the $\mathrm{pH}$ value to 8.5 boosted the yield up to $39.6 \mathrm{mg} / \mathrm{ml}$ (Basak and Majumdar, 1973). In Pandey et al. (2005), different carbon and nitrogen sources were tested for culturing a S. kanamyceticus M27 strain and it was found that using dextrose (chemically identical to glucose) as carbon source and $\mathrm{NH}_{4} \mathrm{H}_{2} \mathrm{PO}_{4}$ (ammonium dihydrogen phosphate) as nitrogen source gave the highest antibiotic production. Thus, the $M$. aurantiaca sp.01 strain of the present study may also increase its kanamycin yield if the culture conditions are adjusted. The $M$. aurantiaca species could potentially be an alternate source of kanamycin. Further study on this is needed.

Another observation is that although the biosynthetic potential of an interested actinobacterial strain is revealed by genomic analysis, expressions of those potentially producible secondary metabolites depend highly on the culture conditions, making many gene clusters remain silent under regular laboratory fermentation conditions. In fact, researches in finding different methods such as homologous and heterologous expression, metabolism remodeling, and metabolic engineering to activate these cryptic genes are very active (Ochi, 2017). Therefore, the present findings also provide evidence for the needs of similar future studies. The close interaction between microbial genomics, biosynthetic logic, and natural product metabolomics is critical not only to the clarification of the structures of new chemical entities but also to the final interpretation of genome sequences.

\section{CONCLUSION}

In conclusion, results of the $M$. aurantiaca $\mathrm{sp} .01$ indicated that this rare actinobacteria species was a potential source for bioactive secondary metabolite production. Genome scanning revealed that at least five antibiotics (similarity more than $15 \%$ in the PKS pathway) could be potentially produced from this strain, and kanamycin was confirmed producible in the fermentation experiment by guided MS/MS analysis. The present findings testified that rare actinobacteria from mangroves are important candidates for future pharmaceutical exploration, as they are relatively unexploited, but indeed contain huge biosynthetic potential. Meanwhile, the approach of combining genome mining and guided MS/MS analysis has been shown to be an efficient and effective way to explore the biosynthetic potential of any interested rare actinobacteria under laboratory culture conditions.

\section{DATA AVAILABILITY STATEMENT}

The datasets presented in this study can be found in online repositories. The names of the repository/repositories and accession number(s) can be found in the article/Supplementary Material. 


\section{AUTHOR CONTRIBUTIONS}

DH, CS, KM, and SL conceived the experiments. DH, CS, and SL undertook sampling work. DH, TJ, and GF conducted the experiment. DH, KM, and SL analyzed the results. DH, KM, KL, and SL wrote the manuscript. All authors read and approved the final manuscript.

\section{FUNDING}

This study was supported by the International S\&T Cooperation (2016YFE0122000), the Macau Science and Technology

\section{REFERENCES}

Ansari, K. G. M. T., Manokaran, S., Raja, S., Lyla, P. S., and Khan, S. A. (2014). Interaction of free-living marine nematodes in the artificial mangrove environment (southeast coast of India). Environ. Monit. Assess 186, 293-305. doi: 10.1007/s10661-013-3374-1

Ansari, M. Z., Yadav, G., Gokhale, R. S., and Mohanty, D. (2004). NRPS-PKS: a knowledge-based resource for analysis of NRPS/PKS megasynthases. Nucleic Acids Res. 32(Suppl._2), W405-W413.

Antal, N., Fiedler, H. P., Stackebrandt, E., Beil, W., Ströch, K., and Zeeck, A. (2005). Retymicin, galtamycin B, saquayamycin Z and ribofuranosyllumichrome, novel secondary metabolites from Micromonospora sp. Tü 6368. J. Antibiot. 58, 103-110. doi: 10.1038/ja.2005.13

Ara, I., Paramaswari, S., and Vikineswary, S. (2002). Diversity of Micromonospora in Malaysian mangrove rhizosphere soils. Malays. J. Pathol. 21, 51-59.

Azman, A. S., Othman, I., Svelu, S., Chan, K. G., and Lee, L. H. (2015). Mangrove rare actinobacteria: taxonomy, natural compound, and discovery of bioactivity. Front. Microbiol. 6:856. doi: 10.3389/fmicb.2015.00856

Bachmann, B. O., Van, L., Steven, G., and Baltz, R. H. (2014). Microbial genome mining for accelerated natural products discovery: is a renaissance in the making? J. Ind. Microbiol. Biotechnol. 41, 175-184. doi: 10.1007/s10295-013$1389-9$

Basak, K., and Majumdar, S. K. (1973). Utilization of carbon and nitrogen sources by Streptomyces kanamyceticus for kanamycin production. Antimicrob. Agents Chemother. 4, 6-10. doi: 10.1128/aac.4.1.6

Cao, X., Diao, M., Zhang, B., Liu, H., Wang, S., and Meng, Y. (2017). Spatial distribution of vanadium and microbial community responses in surface soil of Panzhihua mining and smelting area, China. Chemosphere 183, 9-17. doi: 10.1016/j.chemosphere.2017.05.092

Carro, L., Spröer, C., Alonso, P., and Trujillo, M. E. (2012). Diversity of Micromonospora strains isolated from nitrogen fixing nodules and rhizosphere of Pisum sativum analyzed by multilocus sequence analysis. Syst. Appl. Microbiol. 35, 73-80. doi: 10.1016/j.syapm.2011. 11.003

Challis, G. L. (2014). Exploitation of the Streptomyces coelicolor A3 (2) genome sequence for discovery of new natural products and biosynthetic pathways. J. Ind. Microbiol. Biotechnol. 41, 219-232. doi: 10.1007/s10295-013-1383-2

Cooper, R. M., Tsimring, L., and Hasty, J. (2017). Inter-species population dynamics enhance microbial horizontal gene transfer and spread of antibiotic resistance. eLife 6:e25950.

Cortes, J., Haydock, S. F., Roberts, G. A., Bevitt, D. J., and Leadlay, P. F. (1990). An unusually large multifunctional polypeptide in the erythromycin-producing polyketide synthase of Saccharopolyspora erythraea. Nature 348, 176-178. doi: $10.1038 / 348176 \mathrm{a} 0$

Darken, M. A., Berenson, H., Shirk, R. J., and Sjolander, N. O. (1960). Production of tetracycline by Streptomyces aureofaciens in synthetic media. Appl. Microbiol. 8, 46-51. doi: 10.1128/aem.8.1.46-51.1960

Demain, A. L. (2014). Importance of microbial natural products and the need to revitalize their discovery. J. Ind. Microbiol. Biotechnol. 41, 185-201. doi: $10.1007 / \mathrm{s} 10295-013-1325-\mathrm{z}$
Development Fund (FDCT), and the Ministry of Science and Technology of China (MOST) joint funding scheme (Ref. No. FDCT 017/2015/AMJ) and Research Committee, University of Macau [MYRG2016-00056-FST, MYRG201500182-ICMS-QRCM, MYRG139(Y1-L4)-ICMS12-LMY, and MYRG2016-00129-ICMS-QRCM].

\section{SUPPLEMENTARY MATERIAL}

The Supplementary Material for this article can be found online at: https://www.frontiersin.org/articles/10.3389/fmicb. 2020.01540/full\#supplementary-material

Dhakal, D., Pokhrel, A. R., Shrestha, B., and Sohng, J. K. (2017). Marine rare actinobacteria: isolation, characterization, and strategies for harnessing bioactive compounds. Front. Microbiol. 8:1106. doi: 10.3389/fmicb.2017.01106

Dickschat, J. S., Bruns, H., and Riclea, R. (2011). Novel fatty acid methyl esters from the actinomycete Micromonospora aurantiaca. Beilstein J. Organ. Chem. 7, 1697-1712. doi: 10.3762/bjoc.7.200

Finan, T. M. (2017). The divided bacterial genome: structure, function, and evolution. Microbiol. Mol. Biol. Rev. 81:e00019-17.

Hu, D., Chen, Y., Sun, C., Jin, T., Fan, G., Liao, Q., et al. (2018). Genome guided investigation of antibiotics producing actinomycetales strain isolated from a Macau mangrove ecosystem. Sci. Rep. 8, 1-12.

Hu, D., Gao, C., Sun, C., Jin, T., Fan, G., Mok, K. M., et al. (2019). Genomeguided and mass spectrometry investigation of natural products produced by a potential new actinobacterial strain isolated from a mangrove ecosystem in Futian, Shenzhen, China. Sci. Rep. 9, 1-12.

Huang, C., Leung, R. K.-K., Guo, M., Tuo, L., Guo, L., Yew, W. W., et al. (2016). Genome-guided investigation of antibiotic substances produced by Allosalinactinospora lopnorensis CA15-2T from Lop Nor region, China. Sci. Rep. 6:20667.

Ikeda, H., Ishikawa, J., Hanamoto, A., Shinose, M., Kikuchi, H., Shiba, T., et al. (2003). Complete genome sequence and comparative analysis of the industrial microorganism Streptomyces avermitilis. Nat. Biotechnol. 21, 526-531. doi: $10.1038 /$ nbt 820

Ishii, S. (2019). Ecology of pathogens and antibiotic-resistant bacteria in environments: challenges and opportunities. Microb. Environ. 34, 1-4. doi: 10.1264/jsme2.me3401rh

Jose, P. A., and Jebakumar, S. R. D. (2013). Non-streptomycete actinomycetes nourish the current microbial antibiotic drug discovery. Front. Microbiol. 4:240. doi: $10.3389 /$ fmicb. 2013.00240

Jung, H. M., Kim, S. Y., Moon, H. J., Oh, D. K., and Lee, J. K. (2007). Optimization of culture conditions and scale-up to pilot and plant scales for vancomycin production by Amycolatopsis orientalis. Appl. Microbiol. Biotechnol. 77, 789795. doi: 10.1007/s00253-007-1221-4

Katz, L., and Baltz, R. H. (2016). Natural product discovery: past, present, and future. J. Ind. Microbiol. Biotechnol. 43, 155-176. doi: 10.1007/s10295-0151723-5

Kersten, R. D., Yang, Y. L., Xu, Y., Cimermancic, P., Nam, S. J., Fenical, W., et al. (2011). A mass spectrometry-guided genome mining approach for natural product peptidogenomics. Nat. Chem. Biol. 7, 794-802. doi: 10.1038/nchembio. 684

Kim, H. J., Hwang, I. S., Kim, B. S., and Hwang, B. K. (2006). Isolation and in vitro and in vivo antifungal activity of phenylacetic acid produced by Micromonospora aurantiaca strain JK-1. Plant Pathol. J. 22, 75-89. doi: 10.5423/ ppj.2006.22.1.075

Kim, M., Oh, H., Park, S., and Chun, J. (2014). Towards a taxonomic coherence between average nucleotide identity and 16S rRNA gene sequence similarity for species demarcation of prokaryotes. Int. J. Syst. Evol. Microbiol. 64, 346-351. doi: 10.1099/ijs.0.059774-0

Koolen, H. H. F., Soares, E. R., da Silva, F. M. A., Lima de Souza, A. Q., de Medeiros, L. S., Filho, E. R., et al. (2012). An antimicrobial diketopiperazine alkaloid 
and co-metabolites from an endophytic strain of Gliocladium isolated from Strychnos cf. toxifera. Nat. Prod. Res. 26, 2013-2019. doi: 10.1080/14786419. 2011.639070

Koonin, E. V. (2016). Horizontal gene transfer: essentiality and evolvability in prokaryotes, and roles in evolutionary transitions. F1000Research 5:1805. doi: 10.12688/f1000research.8737.1

Laureti, L., Song, L., Huang, S., Corre, C., Leblond, P., Challis, G. L., et al. (2011). Identification of a bioactive 51-membered macrolide complex by activation of a silent polyketide synthase in Streptomyces ambofaciens. Proc. Natl. Acad. Sci. U.S.A. 108, 6258-6263. doi: 10.1073/pnas.1019077108

Manivasagan, P., Venkatesan, J., Sivakumar, K., and Kim, S. (2015). Marine Actinobacterial Metabolites and their Pharmaceutical Potential. Cham: Springer, 1371-1386.

Mao, D. P., Zhou, Q., Chen, C. Y., and Quan, Z. X. (2012). Coverage evaluation of universal bacterial primers using the metagenomic datasets. BMC Microbiol. 12:66. doi: 10.1186/1471-2180-12-66

Matsumoto, A., and Takahashi, Y. (2017). Endophytic actinomycetes: promising source of novel bioactive compounds. J. Antibiot. 70, 514-519. doi: 10.1038/ja. 2017.20

Molnar, I., Schupp, T., Ono, M., Zirkle, R. E., Milnamow, M., Nowak-Thompson, B., et al. (2000). The biosynthetic gene cluster for the microtubule-stabilizing agents epothilones A and B from Sorangium cellulosum So ce90. Chem. Biol. 7, 97-109. doi: 10.1016/s1074-5521(00)00075-2

Ochi, K. (2017). Insights into microbial cryptic gene activation and strain improvement: principle, application and technical aspects. J. Antibiot. 70, 25-40. doi: 10.1038/ja.2016.82

Ômura, S., Ikeda, H., Ishikawa, J., Hanamoto, A., Takahashi, C., Shinose, M., et al. (2001). Genome sequence of an industrial microorganism Streptomyces avermitilis: deducing the ability of producing secondary metabolites. Proc. Natl. Acad. Sci. U.S.A. 98, 12215-12220. doi: 10.1073/pnas.211433198

Othoum, G., Bougouffa, S., Bokhari, A., Lafi, F., Gojobori, T., Hirt, H., et al. (2019). Mining biosynthetic gene clusters in Virgibacillus genomes. BMC Genomics 20:696. doi: 10.1186/s12864-019-6065-7

Pandey, A., Shukla, A., and Majumdar, S. K. (2005). Utilization of carbon and nitrogen sources by Streptomyces kanamyceticus M 27 for the production of an Anti-bacterial antibiotic. Afr. J. Biotechnol. 4, 909-910.

Prieto-Davó, A., Fenical, W., and Jensen, P. R. (2008). Comparative actinomycete diversity in marine sediments. Aquat. Microb. Ecol. 52, 1-11. doi: 10.3354/ ame 01211

Qian, P., Li, Z., Xu, Y., Li, Y., and Fusetani, N. (2015). Mini-review: marine natural products and their synthetic analogs as antifouling compounds: 2009-2014. Biofouling 31, 101-122. doi: 10.1080/08927014.2014.997226

Raja, A., and Prabakarana, P. (2011). Actinomycetes and drug-an overview. Am. J. Drug Discov. Dev. 1, 75-84. doi: 10.3923/ajdd.2011.75.84

Sanchez, S., and Demain, A. L. (2015). Antibiotics: Current Innovations and Future Trends. Norfolk: Caister Academic Press.

Schatz, A., Bugle, E., and Waksman, S. A. (1944). Streptomycin, a substance exhibiting antibiotic activity against gram-positive and gram-negative bacteria. Proc. Soc. Exp. Biol. Med. 55, 66-69. doi: 10.3181/00379727-55-14461

Sikora, A. E., Tehan, R., and McPhail, K. (2018). Utilization of Vibrio cholerae as a model organism to screen natural product libraries for identification of new antibiotics. In Vibrio Cholerae 1839, 135-146. doi: 10.1007/978-1-4939-86859_12

Sripreechasak, P., Matsumoto, A., Suwanborirux, K., Inahashi, Y., Shiomi, K., Tanasupawat, S., et al. (2013). Streptomyces siamensis sp. nov., and Streptomyces similanensis sp. nov., isolated from Thai soils. J. Antibiot. 66:633. doi: 10.1038/ ja.2013.60

Subramani, R., and Aalbersberg, W. (2013). Culturable rare actinomycetes: diversity, isolation and marine natural product discovery. Appl. Microbiol. Biotechnol. 97, 9291-9321. doi: 10.1007/s00253-013-5229-7
Tiwari, K., and Gupta, R. K. (2012). Rare actinomycetes: a potential storehouse for novel antibiotics. Crit. Rev. Biotechnol. 32, 108-132. doi: 10.3109/07388551. 2011.562482

Turnipseed, S. B., Clark, S. B., Karbiwnyk, C. M., Andersen, W. C., Miller, K. E., and Madson, M. R. (2009). Analysis of aminoglycoside residues in bovine milk by liquid chromatography electrospray ion trap mass spectrometry after derivatization with phenyl isocyanate. J. Chromatogr. B 877, 1487-1493. doi: 10.1016/j.jchromb.2009.03.025

Udwary, D. W., Zeigler, L., Asolkar, R. N., Singan, V., Lapidus, A., Fenical, W., et al. (2007). Genome sequencing reveals complex secondary metabolome in the marine actinomycete Salinispora tropica. Proc. Natl. Acad. Sci. U.S.A. 104, 10376-10381. doi: 10.1073/pnas.0700962104

Umezawa, H. (1958). Kanamycin: its discovery. Ann. N. Y. Acad. Sci. 76, 20-26. doi: 10.1111/j.1749-6632.1958.tb54688.x

Umezawa, H., Ueda, M., Maeda, K., Yagishita, K., Kondo, S., Okami, Y., et al. (1957). Production and isolation of a new antibiotic: kanamycin. J. Antibiot. $10,181-188$.

Wang, S., Zhang, B., Li, T., Li, Z., and Fu, J. (2020). Soil vanadium (V)-reducing related bacteria drive community response to vanadium pollution from a smelting plant over multiple gradients. Environ. Int. 138:105630. doi: 10.1016/ j.envint.2020.105630

Weinstein, M. J., Luedemann, G. M., Oden, E. M., Wagman, G. H., Rosselet, J. P., Marquez, J. A., et al. (1963). Gentamicin, 1 a new antibiotic complex from Micromonospora. J. Med. Chem. 6, 463-464. doi: 10.1021/jm003 $40 \mathrm{a} 034$

Wright, G. D. (2019). Unlocking the potential of natural products in drug discovery. Microb. Biotechnol. 12, 55-57. doi: 10.1111/1751-7915.13351

Xiao, Y. S., Zhang, B., Zhang, M., Guo, Z. K., Deng, X. Z., Shi, J., et al. (2017). Rifamorpholines A-E, potential antibiotics from locust-associated actinobacteria Amycolatopsis sp. Hca4. Organ. Biomol. Chem. 15, 3909-3916. doi: 10.1039/c7ob00614d

Xu, J. Y., You, D., Leng, P. Q., and Ye, B. C. (2014). Allosteric regulation of a protein acetyltransferase in Micromonospora aurantiaca by the amino acids cysteine and arginine. J. Biol. Chem. 289, 27034-27045. doi: 10.1074/jbc.m114.579078

Xu, L., Li, Q., and Jiang, C. (1996). Diversity of soil actinomycetes in Yunnan, China. Appl. Environ. Microbiol. 62, 244-248. doi: 10.1128/aem.62.1.244-248. 1996

Zhang, B., Cheng, Y., Shi, J., Xing, X., Zhu, Y., Xu, N., et al. (2019a). Insights into interactions between vanadium $(\mathrm{V})$ bio-reduction and pentachlorophenol dechlorination in synthetic groundwater. Chem. Eng. J. 375:121965. doi: 10. 1016/j.cej.2019.121965

Zhang, B., Wang, S., Diao, M., Fu, J., Xie, M., Shi, J., et al. (2019b). Microbial community responses to vanadium distributions in mining geological environments and bioremediation assessment. J. Geophys. Res. Biogeosci. 124, 601-615. doi: 10.1029/2018jg004670

Zhang, X., Wang, J., Wu, Q., Li, L., Wang, Y., and Yang, H. (2019). Determination of kanamycin by high performance liquid chromatography. Molecules 24:1902. doi: 10.3390/molecules24101902

Conflict of Interest: The authors declare that the research was conducted in the absence of any commercial or financial relationships that could be construed as a potential conflict of interest.

Copyright (c) $2020 \mathrm{Hu}$, Sun, Jin, Fan, Mok, Li and Lee. This is an open-access article distributed under the terms of the Creative Commons Attribution License (CC BY). The use, distribution or reproduction in other forums is permitted, provided the original author(s) and the copyright owner(s) are credited and that the original publication in this journal is cited, in accordance with accepted academic practice. No use, distribution or reproduction is permitted which does not comply with these terms. 